\title{
Carcinoma espinocelular em assoalho bucal: relato de caso
}

\author{
Squamous cell carcinoma in the mouth floor: clinical case \\ Carcinoma de células escamosas de cavidad bucal: reporte de caso \\ Dannilo Wiklymber Roldao MENDONÇA ${ }^{\mathbf{1}}$ \\ Helinaldo Corrêa da CONCEIÇÃO ${ }^{2}$ \\ Gleicy Gomes de LIMA ${ }^{3}$ \\ Maria de Fátima ARAÚjO ${ }^{4}$ \\ Lioney Nobre CABRAL ${ }^{5}$ \\ Tiago Novaes PINHEIRO 6 \\ Cirurgião Dentista da UBS Fluvial Estácio Oduvaldo (Mastro), do Município de Autazes-AM, 69240-000, Brasil. \\ ${ }^{2}$ Cirurgião Dentista da UBS Fluvial do Município de Manicoré-AM, 69280-000, Brasil \\ ${ }^{3}$ Acadêmica do Curso de Graduação em Odontologia, Escola Superior de Ciências da Saúde, Universidade do Estado do Amazonas (UEA) \\ 69065-001 Manaus-AM, Brasil \\ ${ }^{4}$ Doutoranda em Saúde Coletiva, Instituto de Medicina Social, Universidade do Estado do Rio de Janeiro, IMS-UERJ, 20550-013 Rio de Janeiro - RJ, Brasil \\ ${ }^{5}$ Doutor em Biotecnologia pela Universidade Federal do Amazonas (UFAM). Professor da Escola Superior de Ciências da Saúde, \\ Universidade do Estado do Amazonas (UEA) 69065-001 Manaus-AM, Brasil \\ ${ }^{6}$ Mestre e Doutor em Patologia Bucal pela Faculdade de Odontologia de Bauru, Universidade de São Paulo (USP), Professor da Escola Superior de Ciências da Saúde, \\ Universidade do Estado do Amazonas (UEA) 69065-001 Manaus-AM, Brasil
}

\section{Resumo}

O câncer de boca representa um problema de saúde pública em todo o mundo, das neoplasias malignas que podem afetar a cavidade oral, o carcinoma espinocelular (CEC), destaca-se por cerca de $90 \%$ de todas as lesões malignas diagnosticadas na boca. Descrever o diagnóstico de um caso de (CEC) em região de assoalho bucal em um paciente tabagista. Paciente com 43 anos de idade, sexo masculino, melanoderma, garimpeiro, cor parda, compareceu ao atendimento na Policlínica Odontológica da Universidade do Estado do Amazonas (UEA) para exame estomatológico de rotina. Durante a anamnese o paciente relatou ser tabagista e etilista há mais de 30 anos e fazia uso de cigarros de maconha frequentemente. Ainda através da anamnese, soube-se que o paciente foi exposto por muito tempo à luz solar quando o mesmo trabalhava como garimpeiro. Mencionou também sentir bastante dor e ardência na região da lesão. Diante das informações clínicas colhidas, chegou-se à hipótese diagnóstica de (CEC). O diagnóstico foi confirmado por meio de biopsia incisional e exame histopatológico, com posterior encaminhamento para tratamento médico junto ao centro de controle de oncologia de Manaus (AM). Compete ao cirurgião-dentista diagnosticar e tratar em conjunto com outras especialidades, já que o diagnóstico precoce desta neoplasia torna-se essencial.

Descritores: Neoplasias Bucais; Carcinoma de Células Escamosas; Soalho Bucal.

\section{Abstract}

Mouth cancer represents a public health problem worldwide, of malignant neoplasms that can affect the oral cavity, squamous cell carcinoma (SCC), stands out for about $90 \%$ of all malignancies diagnosed in the mouth. To describe the diagnosis of a case of (SCC) in a buccal floor region in a smoker. The patient M.F.A. 43 years old male, melanoderma, garimpeiro, brown, attended the service in the UEA polyclinic for routine stomatological examination. During the anamnesis the patient reported being a smoker and an alcoholic for more than 30 years and used marijuana cigarettes frequently. Still through the anamnesis, it was learned that the patient was exposed for a long time to sunlight when he worked as a prospector. He also mentioned feeling a lot of pain and burning in the area of the injury. In view of the clinical information collected, the diagnostic hypothesis (SCC) was reached. The diagnosis was confirmed by means of an incisional biopsy and histopathological examination, with subsequent referral for medical treatment to the oncology control center-Manaus. Compete to the dental surgeon to diagnose and treat them together with other specialties, since the early diagnosis of this malignancy becomes essential.

Descriptors: Mouth Neoplasms; Carcinoma, Squamous Cell; Mouth Floor.

\section{Resumen}

El cáncer de boca representa un problema de salud pública en todo el mundo, debido a las neoplasias malignas que pueden afectar la cavidad oral, el carcinoma de células escamosas (SCC) se destaca en aproximadamente el $90 \%$ de todas las lesiones malignas diagnosticadas en la boca. Describo el diagnóstico de un caso de CPB en la región del piso oral en un paciente fumador. El paciente M.F.A. Varón de 43 años, melanoderma, minero de oro, color marrón, asistió a la atención en el policlínico UEA para un examen estomatológico de rutina. Durante la anamnesis, el paciente informó ser fumador y alcohólico por más de 30 años y usó cigarrillos de marihuana con frecuencia. Aún a través de la anamnesis, se sabía que el paciente estuvo expuesto a la luz solar durante mucho tiempo cuando trabajaba como buscador de oro. También mencionó sentir mucho dolor y ardor en el área de la lesión. En vista de la información clínica recopilada, se llegó a la hipótesis diagnóstica de (CPB). El diagnóstico se confirmó mediante la toma de biopsia de la lesión y examen histopatológico, con posterior encaminado del paciente para tratamiento médico en el centro de control oncológico de Manaus (AM). Depende del dentista diagnosticar y tratar junto con otras especialidades, ya que el diagnóstico temprano de esta neoplasia se vuelve esencial.

Descriptores: Neoplasias de la Boca; Carcinoma de Células Escamosas; Suelo de la Boca.

\section{INTRODUÇÃO}

A estimativa mundial mostra que, em 2012, ocorreram 14,1 milhões de casos novos de câncer e 8,2 milhões de óbitos. De modo geral, as maiores taxas de incidências foram observadas nos países desenvolvidos (América do Norte, Europa Ocidental, Japão, Coréia do Sul, Austrália e Nova Zelândia). Para o Brasil, estimam-se 11.200 casos novos de câncer da cavidade oral em homens e $3.500 \mathrm{em}$ mulheres para cada ano do biênio 2018-2019. Sendo a classificação por região, o câncer da cavidade oral em homens na região Norte (3,59/100 mil), ocupa a sexta posição. Para as mulheres da região Norte $\left(1,78 / 100\right.$ mil), é o $12^{\circ}$ mais frequente ${ }^{1,2}$.
O carcinoma espinocelular (CEC), também denominado carcinoma epidermóide, carcinoma escamocelular e carcinoma de células escamosas (CCE), é uma neoplasia maligna mais comum de cavidade oral, representando $90 \%$ a $96 \%$ dos cânceres bucais, sendo constituído pela multiplicação atípicade células espinhosas e podendo surgir em qualquer sítio do tegumento ${ }^{3}$.

A sua incidência varia de acordo com idade, gênero, hábito, ocupação, grupos étnicos e localização geográfica. Embora possa ocorrer em qualquer parte da cavidade oral, certas áreas são mais frequentemente afetadas do que outras como, lábio 
inferior que representa entre $25 \%$ e $40 \%$ dos carcinomas bucais e assoalho bucal sendo a segunda localização intrabucal mais comum dos carcinomas espinocelular representando de $15 \%$ a $20 \%$ dos $\operatorname{casos}^{4-6}$.

O CEC oral é reconhecidamente a neoplasia que atinge preferencialmente os paciente do gênero masculino e a faixa etária entre 50 e 70 anos especialmente naqueles que são tabagistas $\mathrm{e}$ etilistas. ${ }^{6,7}$ Além destes, a exposição solar excessiva sem a devida proteção ao longo dos anos constitui-se em um considerável fator de risco para o câncer de boca, especialmente o de lábio ${ }^{8-11}$.

Um fator associado ao câncer de boca é o papilomavirus humano (HPV) de alto risco (como HPV 16, 18 e 31), em associação a outros fatores etiológicos. Embora estudos mostrem uma associação desse vírus com o CEC de boca, e até com lesões potencialmente malignas, ainda não foi demonstrada realmente a participação desse vírus na carcinogênese de boca, seguindo os postulados de Koch, como por exemplo, uma infecção viral precedendo o carcinoma ou uma associação epidemiológica. Segundo Koch em 1999, existe uma alta taxa de perda de heterozigozidade em $3 p$, 4q e 11q13, assim como a mutação em p53 também mostrou se mais prevalente em indivíduos fumantes com carcinoma espinocelular de boca $^{12-14}$.

Há também evidências que hábitos alimentares com baixos padrões nutricionais associados a estilo de vida podem ser fatores coadjuvantes na etiologia do câncer bucal ${ }^{15,16}$. Alguns alimentos podem estar associados à redução do risco para essas lesões, como por exemplo: frutas e vegetais, além da associação feijão com arroz ${ }^{17}$.

$\mathrm{O}$ tratamento do câncer de cabeça e pescoço pode ser realizado através das seguintes modalidades terapêuticas: cirurgia, radioterapia, quimioterapia ou combinação destas, dependendo do diagnóstico definitivo, estadiamento e localização da lesão ${ }^{18} \cdot E m$ relação ao prognóstico, vários fatores devem ser considerados: graduação histopatológica e localização do tumor, idade avançada, estado de saúde geral e do sistema imunológico do paciente ${ }^{19}$.

A divulgação deste caso clínico é de grande importância no meio científico e social, servindo como base para direcionar no diagnóstico e tratamento em situações semelhantes, visto que trabalhos versando sobre carcinoma espinocelular são importantíssimos na literatura científica nacional e internacional.

\section{CASO CLÍNICO}

Paciente M.F.A., gênero masculino, 43 anos, melanoderma, solteiro, procedente do município de Manaus, Amazonas, apresentou-se em consulta à Policlínica Odontológica da Universidade do Estado do Amazonas (UEA) no dia 31 de março de 2014, queixando-se de um "buraco embaixo da língua".
Durante a anamnese, o paciente relatou ser tabagista e etilista há mais de 30 anos e fazia uso de cigarros de maconha frequentemente. Ainda através da anamnese soube-se que o paciente foi exposto por muito tempo à luz solar quando o mesmo trabalhava como garimpeiro. Mencionou também sentir bastante dor e ardência na região da lesão. Diante das informações clínicas colhidas, chegou-se à hipótese diagnóstica de Carcinoma espinocelular (CEC). Ao ser realizado o exame extrabucal, constatou a presença de umlinfonodo cervical esquerdo endurecido, fixo, de superfície irregular eassintomático ao toque (Figura 1), e também um linfonodo na região submandibular esquerdo endurecido, móvel e assintomático (Figura 2). Em uma visão frontal é possível ver nitidamente o linfonodo submandibular e o cervical esquerdo (Figura 3).

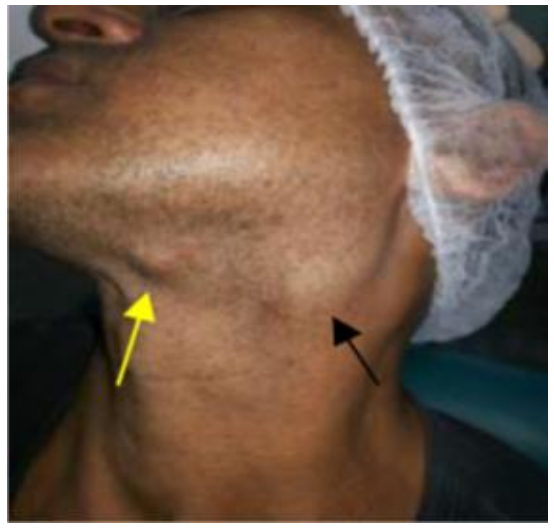

Figura 1: Seta preta indica linfonodo cervical. Seta amarela indica linfonodo submandibular

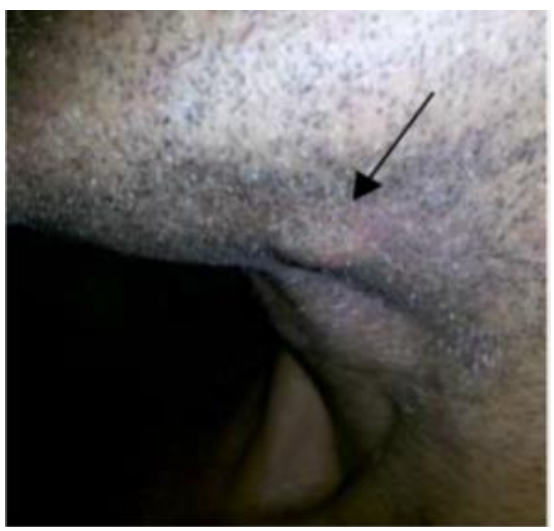

Figura 2: Linfonodo submandibular.

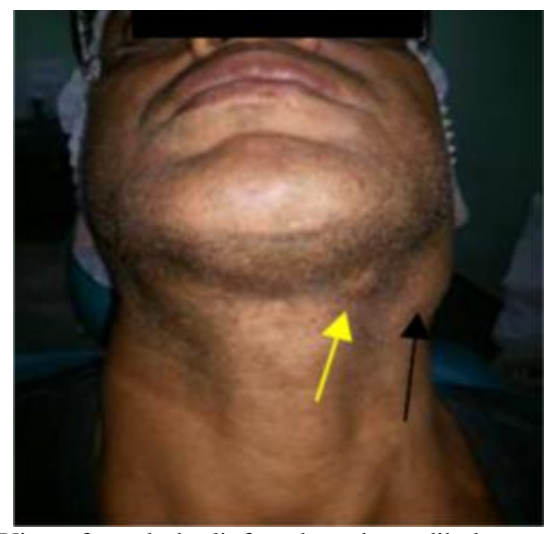

Figura 3. Visão frontal do linfonodo submandibular seta amarela e cervical seta preta. 
Ao exame clínico intrabucal, observou-se uma lesão úlcera vegetativa com bordas irregulares, mal definidas, aspecto contornado de base séssil com endurecimento periférico. Observou-se também aspecto queratinizado ao redor da úlcera, localizada no assoalho bucal esquerdo e se estendendo em direção a linha média a base da língua, com maior diâmetro de $7 \mathrm{~mm}$ (Figura 4). Na tomografia foi observada discreta imagem hipodensa no corpomandibular na região de canino inferior esquerdo se estendendo para a regiãode molares inferiores (Figura 5). Para realização da biópsia incisional foram solicitados os seguintes exames complementares: coagulograma, hemograma completo e teste de glicemia. Para serem entregues antes da realização do procedimento cirúrgico. Após uma semana o paciente retorna à Policlínica Odontológica da Universidade do Estado do Amazonas-UEA para realização da biópsia incisional, que consiste na remoção de uma parte da lesão. A cirurgia foi realizada com anestesia local, utilizando o anestésico Articaína 4\%+ Epinefrina (Adrenalina) 1:100.000. Foi realizada uma incisão contornando uma parte da lesão (Figura 6), e em seguida, foi feita a remoção parcial da mesma para posterior exame histopatológico (Figura 7). Aspecto clínico após lesão ser suturada (Figura 8).

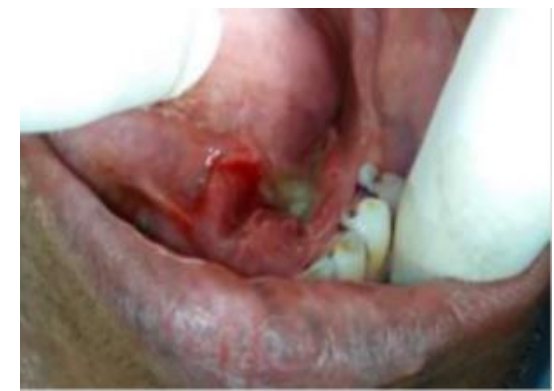

Figura 4. Aspecto clínico da lesão em assoalho bucal.

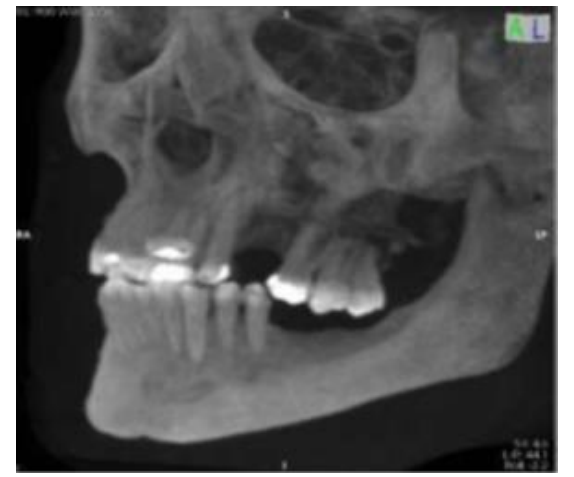

Figura 5: Tomagrafia computadorizada

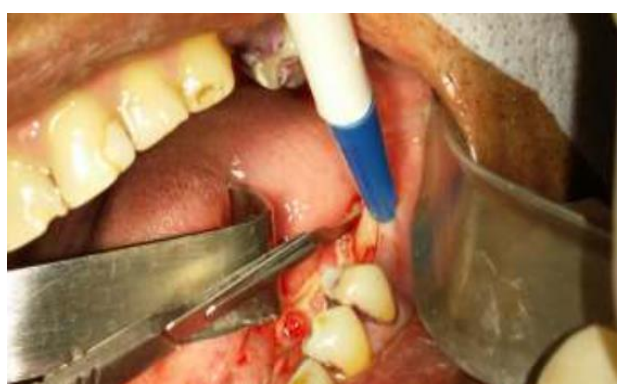

Figura 6: Incisão contornando uma parte da lesão.

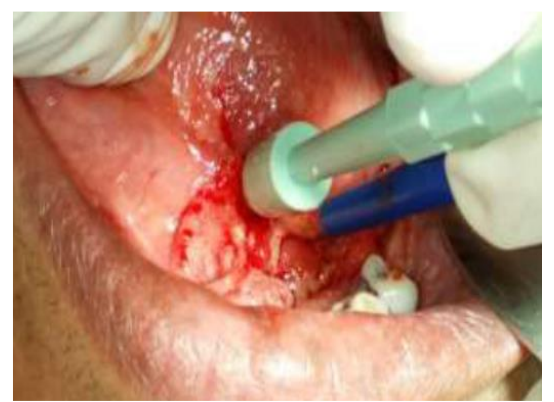

Figura 7: Remoção parcial da lesão com punch.

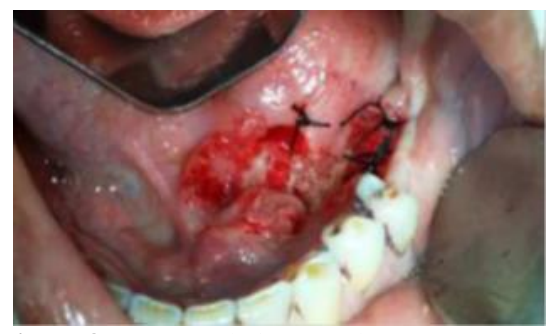

Figura 8: Aspecto clínico após lesão ser suturada

Em uma visão macroscópica, mostrou-se dois fragmentos de tecido mole consistência firme de forma e superfície irregular de cor acastanhado medindo1,3x 0,5x 0,2 cm (Figura 9). A peça removida durante a biópsia foi acondicionada em um frasco contendo formol a $10 \%$, devidamente identificado, e encaminhado posteriormente para uma análise histopatológica no laboratório (Figura 10). No preenchimento da solicitação do exame microscópico, foi colocado como possível hipótese diagnóstica Carcinoma espinocelular (CEC). O laudo histopatológico revelou mucosa parcialmente revestida por epitélio estratificado pavimentoso paraqueratinizado. Observam-se ainda hipercromatismo e pleomorfismo celular em camadas basal, parabasal e espinhosa do revestimento epitelial, além de intensa exocitose leucocitária (Figura 11).

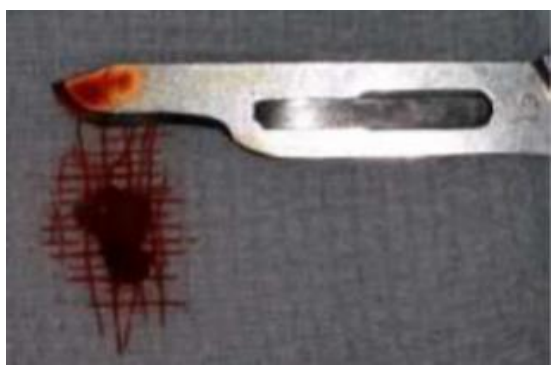

Figura 9: Remoção parcial da lesão.

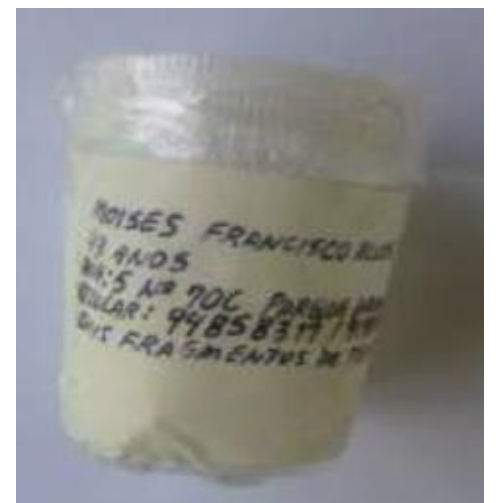

Figura 10: Fragmento de tecido mole encaminhado para biópsia. 


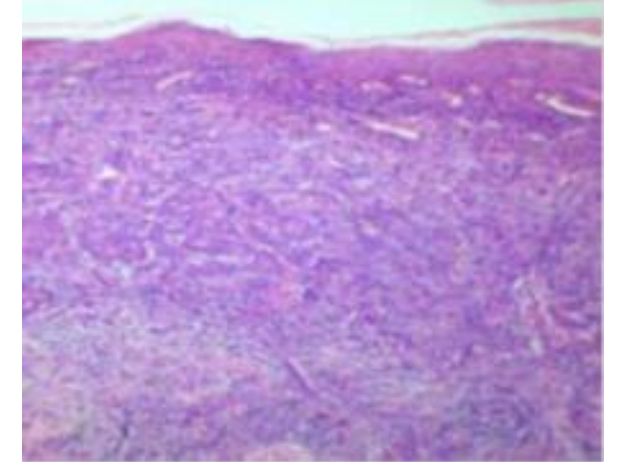

Figura 11: Epitélio estratificado pavimentoso paraqueratinizado.

Em lâmina própria, composta por tecido conjuntivo denso não modelado, há formação de ilhotas e cordões de células de fenótipo epitelial, com alto grau de pleomorfismo, ora exibindo núcleos volumosos com nucléolos evidentes, perda de proporção núcleo-citoplasma e núcleo-nucléolo, ora exibindo intenso hipercromatismo e anisocariose (Figura 12). Nestes grupos celulares podem ser notadas inúmeras figuras mitóticas, muitas das quais atípicas. Em profundidade podem ser notadas áreas de invasão muscular e infiltração perivascular. Há intenso infiltrado inflamatório do tipo mononuclear, frequentemente margeando as ilhotas e cordões epiteliais. Completa o quadro a presença de glândulas salivares seromucosas e áreas hemorrágicas (Figura13). De acordo com essas características, o diagnóstico definitivo foi de Carcinoma espinocelular. Uma semana após o procedimento cirúrgico, o paciente voltou à Policlínica Odontológica da UEA para retirada da sutura (Figura 14). Frente a este resultado, o paciente foi encaminhado para tratamento na Fundação Centro de Controle de Oncologia do Estado do AmazonasFCECON. Devido o paciente apresentar uma lesão em estágio avançado, o mesmo foi submetido à radioterapia e quimioterapia.

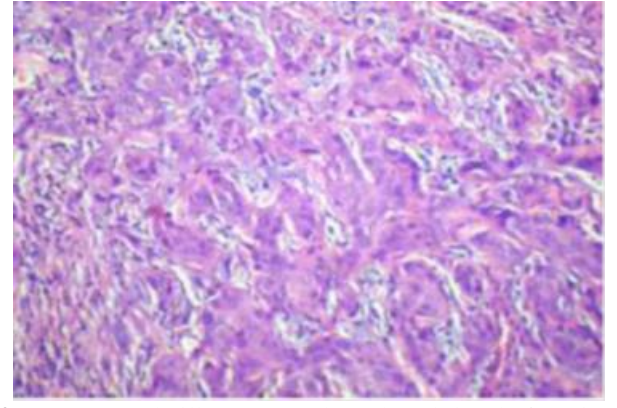

Figura 12: Formação de ilhotas e cordões de células de fenótipo epitelial.

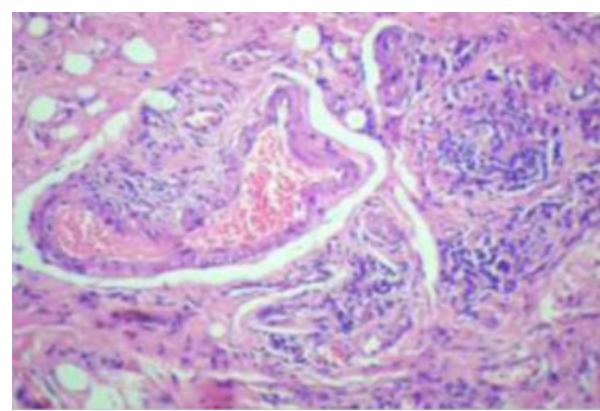

Figura 13: Glândulas salivares seromucosas.

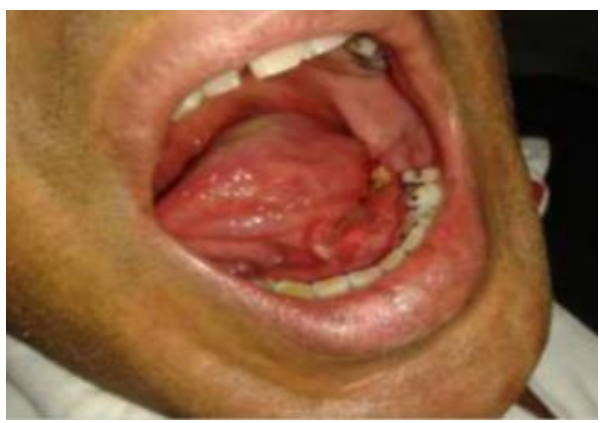

Figura 14: Remoção de sutura 7 dias após o procedimento cirúrgico.

DISCUSSÃO

A maioria dos tumores malignos da boca é representada por CEC, sendo mais comum em homens com idade superior a $45 \operatorname{anos}^{20}$. Não é um trabalho concordante com o caso clinico visto que o paciente do relato tem idade de 43 anos. Porém é concordante com o trabalho de Sassi et al. ${ }^{21}$ que relataram o de caso de um paciente jovem de apenas 21 anos de idade.

$\mathrm{O}$ aspecto clínico do $\mathrm{CEC}$ de boca parece não apresentar características distintas, qualquer que seja a idade do paciente. A característica clássica da lesão é constituída por úlcera persistente com endurecimento e infiltração periférica, podendo ou não estar associada a vegetações, manchas avermelhadas ou esbranquiçadas, além de linfonodo cervical $^{22}$.

No relato de caso clínico descrito no presente trabalho, observou-se uma lesão úlcera vegetativa com bordas irregulares, mal definidas, aspectos contornados de base séssil com endurecimento periférico, características também presentes foram manchas avermelhadas e esbranquiçadas, bem como linfonodo cervical e submandibular, corroborando os achados de Friedlander et al. ${ }^{22}$.

Os homens tiveram quase 3 vezes mais câncer de boca que as mulheres, ou seja, os autores atribuem isso a maior exposição ao tabagismo e ao etilismo entre os homens ${ }^{23}$. O paciente do relato de caso é homem, durante a anamnese relatou ser tabagista e etilista há mais de 30 anos, além do mais fazia uso de cigarros de maconha, percebe-se por outros estudos que a maconha potencializa os riscos de CEC. A fumaça da maconha acarreta a inalação 3 vezes maior de partículas e a retenção de $33 \%$ maior delas no trato respiratório, em relação a fumaça do cigarro $^{24}$.

Para avaliação e os procedimentos cabíveis. Foram solicitados exames de risco cirúrgico, tomografia de face, pescoço e tórax, para observar extensão da lesão, possível anormalidade maxilomandibular e para ajudar na escolha do tratamento para o paciente ${ }^{25}$.

O relato de caso é concordante, pois para realização da biópsia incisional foram solicitados os seguintes exames complementares: coagulograma, hemograma completo e teste de glicemia. Para serem 
entregues antes da realização do procedimento cirúrgico. Na tomografia foi observada discreta imagem hipodensa no corpomandibular na região de canino inferior esquerdo se estendendo para a região de molares inferiores.

\section{CONSIDERAÇÕES FINAIS}

O exame clínico bucal aprimorado é importantíssimo em todas as consultas, permitindo investigação e diagnóstico de lesões não apontadas como queixa principal. Nos indivíduos de maior risco o exame deve ser sistemático e, sobretudo naqueles com lesões suspeitas de malignidade, devem ser encaminhados à consulta especializada. Um diagnóstico primário é fundamental para promoção de qualidade de vida aos pacientes. Para melhor diagnosticar é essencial à associação de exames complementares de imagem, bem como laboratoriais.

\section{REFERÊNCIAS}

1. Ferlay J, Soerjomataram I, Dikshit R, Eser S, Mathers C, Rebelo $\mathrm{M}$ et al. Cancer incidence and mortality wordwide: sources, methods and major patterns in Globocan 2012. Int J Cancer. 2015; 136(5):E359-386.

2. Instituto Nacional de Câncer Jose Alencar Gomes da Silva. Estimativa 2018: incidência de câncer no Brasil / Coordenação de Prevenção e Vigilância. Rio de Janeiro: INCA, 2017.

3. Dib LL, Sabba LMB, Marques LA, Araújo NS. Fatores prognósticos em carcinoma de bordas de língua: análise clínica e histopatológica. Acta Oncol Bras. 1994;14(2):88-93.

4. Regezi JA, Sciubba JJ, Jordan RCK. Patologia oral: correlações clinicopatológicas. 5. Ed. Rio de Janeiro: Elsevier; 2008.

5. Neville BW, Damm DD, Alen CM, Bouquot JE. Patologia oral e maxilofacial. Rio de Janeiro: Elsevier; 2009.

6. Abreu MAMM, Pimentel DRN, Silva OMP, Blachman IT, Michalany NS, Hirata $\mathrm{CH}$ et al. Squamous cell carcinoma of the lip: assessment of prognostic factors. Rev Bras Otorrinolaringol. 2004;70(6):765-70.

7. Oliveira LR, Ribeiro Silva A, Zucoloto S. Perfil da incidência e da sobrevida de pacientes com carcinoma epidermóide oral em uma população brasileira. J Bras Patol Med Lab. 2006;42(5):385-92.

8. Figuero Ruiz E, Carretero Peláez MA, Cerero Lapiedra R, Esparza Gómez G, Moreno López LA. Effects of the consumption of alcohol in the oral cavity: relationship with oral cancer. Med Oral. 2004;9(1):14-23.

9. Fardin M, Freitas SM, Dedivitis RA, Rapoport A, Denardin OVP, A-Sobrinho J. Fatores de risco no prognóstico do câncer da boca: estudo de 1440 casos. Rev Bras Cirurgia Cabeça Pescoço. 2004; 33(1):27-33.
10. Silva PSL, Leão VML, Scarpel RD. Caracterização da população portadora de câncer de boca e orofaringe atendida no setor de cabeça e pescoço em hospital de referência na cidade de Salvador - BA. Rev CEFAC. 2009;11(3):441-47.

11. Souza LRB, Ferraz KD, Pereira NS, Martins MV. Conhecimento acerca do câncer bucal e atitudes frente à sua etiologia e prevenção em um grupo de horticultores de Teresina (PI). Rev Bras Cancerol. 2012;58(1):31-9.

12. Syrjänen S, Lodi G, von Bultzingslowen I, Aliko A, Arduino P, Campisi G et al. Human papilloma viruses in oral carcinoma and oral potentially malignant disorders: a systematic review. Oral Dis. 2011;17(Suppl 1):58-72.

13. Vidal AKL, Caldas Júnior AF, Mello RJV, Brandão VRA, Rocha GI, Taromaru E. J Bras Patol Med Lab. 2004;40(1):21-6.

14. Sina M, Pedram M, Ghojazadeh M, Kochaki A, Aghbali A. P53 gene codon 72 polymorphism in patients with oral squamous cell carcinoma in the population of northern Iran. Med Oral Patol Oral Cir Bucal. 2014;19(6):e550-55.

15. Marchioni DML, Fisberg RM, Gois Filho JF, Kowalski LP, Carvalho MB, Abrahão $M$ et al. Fatores dietéticos e câncer oral: estudo casocontrole na região metropolitana de São Paulo, Brasil. Cad Saúde Pública. 2007;23(3):553-64.

16. Paiva M, Pileggi C, Nobile CGA, Angelillo IF. Association between fruit and vegetable consumption and oral cancer: a meta-analysis of observational studies. Am J Clin Nutr. 2006; 83(5):1126-34.

17. Brown LM, Moradi T, Gridley G, Plato N, Dosemeci M, Fraumeni Jr JF. Exposures in the paiting trades and paint manufacturing industry and risck among men and women in Sweden. J Occup Environ Med. 2002;44(3):258-64.

18. Silva MFA. Avaliação da condição de saúde bucal e da qualidade de vida em pacientes com câncer de cabeça e pescoço após radioterapia [monografia]. Campina Grande: UEPB; 2012.

19. Coaracy AEV, Lopes FF, Cruz MCFN, Bastos EG. Correlação entre os dados clínicos e histopatológicos dos casos de carcinoma espinocelular oral do Instituto Maranhense de Oncologia Aldenora Bello, em São Luís, MA. J Bras Patol Med Lab. 2008;44(1): 31-5.

20. Iamarron A, Pattanaporn K, Pongsiriwet S, Wanachantararak S, Prapayasatok S,Jittidecharaks $S$ et al. Analysis of 587 cases of oral squamous cell carcinoma in northern Thailand with a focus on young people. Int $\mathbf{J}$ Oral Maxillofac Surg. 2004;33(1):84-8.

21. Sassi LM, Oliveira BV, Pedruzzi PAG, Ramo GHA, Stramandinoli RT, Gugelmin G et al. Carcinoma espinocelular de boca em paciente 
jovem: relato de caso e avaliação dos fatores de risco. RSBO. 2010;7(1):105-9.

22. Friedlander PL, Schantz SP, Shaha AR, Yu G, Shah JP. Squamous cell carcinoma of the tongue in young patients: a matched-pair analysis. Head Neck. 1998;20(5):363-68.

23. Gomes LC, Macena FCS, Ferreira VS, Barreto VR. Revisão de Literatura: câncer de boca diagnóstico e fatores de riscos associados. Rev Interdisciplinar em saúde. 2018;5(4):655-70.

24. Myers JN, Elkins T, Roberts D, Byers RM. Squamous cell carcinoma of the tongue in young adults: increasing incidence and factors that predict treatment outcomes. Otolaryngol Head Neck Surg. 2000;122(1):44-51.

25. Souza DP. Carcinoma espinocelular relacionado ao hábito de mascar tabaco: relato de caso clínico [monografia]. Manaus: Universidade do Estado do Amazonas - UEA; 2017.

\section{CONFLITO DE INTERESSES}

Os autores declaram não haver conflitos de interesse.

\section{AUTOR PARA CORRESPONDENCIA}

Helinaldo Corrêa da Conceição

helinaldouea@gmail.com

Submetido em 22/01/2019

Aceito em 12/03/2019 